Physics

Electricity \& Magnetism fields

Okayama University

Year 1989

\title{
3-D non-linear eddy current analysis using the time-periodic finite element method
}

Takayoshi Nakata

Okayama University

Koji Fujiwara

Okayama University
Norio Takahashi

Okayama University

Akira Ahagon

Okayama University

This paper is posted at eScholarship@OUDIR : Okayama University Digital Information Repository.

http://escholarship.lib.okayama-u.ac.jp/electricity_and_magnetism/4 
3-D NON-LINEAR EDDY CURRENT ANAIYSIS

USING THE TIME-PERIODIC FINITE ELEMENT METHOD

T.Nakat.a, N.Takahashi, K.Fu.jiwara and A.Ahagon

Dept. of Electrical Engineering, Okayama University, Okayama 700 , Japan

\section{ABSTRACT}

The 3-D finite element method using the $A-\phi$ formulation for analyzing time-periodic non-linear magnetic fields with eddy currents has been developed. The CPU time of the new method can be reduced than that of the conventional step-by-step method, because the new method calculates the time-periodic phenomena directly not through the transient phenomena.

\section{INTRODUCTION}

When periodic non-linear fields are analyzed taking into account eddy currents and voltage sources[1], it is not practical to apply the so-called step-by-step nethod 21 to such problems. Because a number of iterations are necessary until periodic solutions are obtained and the very long CPU time is consumed. The improved 2-D finite element method for such problems has already been developed $|3,4|$. The improved method is called the "time-periodic finite element method". In this method, a time-periodic waveforn can be directly calculated not through the transient phenomena by efficiently using the relationship between the vector potentials at the instants $t$ and $t+T / 2$ ('T:period) of the periodic waveform. The matrix equation is constructed in half a period and the vector potentials in half a period are solved at the same time. The CPU time can be reduced compared with the conventional step-by-step method without much increase of the computer storage. This advantage is especially important in 3-D magnetic field analysis.

In this paper, the method is expanded into 3-D analysis of non-linear magnetic fields with eddy currents using the $A-\phi$ formulation. The finite element discretization of the expanded method is described in detail. As an example of application, a loaded transformer is analyzed by using the new method and the conventional step-by-step method, and the CPU times and the computer storages of both methods are compared with each other

\section{METHOD OF ANALYSIS}

\section{$\underline{2.1 \text { Fundamental Equations }}$}

In the $A-\phi$ method, the following equations are discretized in order to analyze $3-D$ magnetic fields with eddy currents[1]:

$$
\begin{aligned}
& \mathbb{G o i}=-\iint_{\Omega} \operatorname{gradNi} \times(v \operatorname{rot} A) d V \\
& +\iint_{\Omega \mathrm{e}} \mathrm{Ni} \sigma\left(\frac{\partial \mathrm{A}}{\partial \mathrm{t}}+\operatorname{grad} \phi\right) d V \\
& -\iint_{\Omega \mathrm{C}} \mathrm{N} \text { i J } \mathrm{dV}=0 \\
& \mathrm{Gdi}=\iint_{\Omega \mathrm{e}} \operatorname{gradNi} \cdot \sigma\left(\frac{\partial A}{\partial t}+\operatorname{grad} \phi\right) d V=0
\end{aligned}
$$

where $A$ and $\phi$ are the magnetic vector potential and the electric scalar potential respectively. Io is the current density in the exciting winding. $v$ and $\sigma$ are the reluctivity and the conductivity respectively. $\mathrm{Ni}$ is the interpolation function. $\Omega$ denotes the analyzed region. $\Omega \mathrm{e}$ and $\Omega \mathrm{c}$ are the region of conductors with eddy currents and that of windings where eddy currents are neglected.

When electrical machines excited from voltage sources are analyzed, not only Eqs. (1) and (2) but also the following equation derived from Kirchhoff's second law should be discretized[1]:

$$
\eta=V_{0}-\frac{d \Psi}{d t}-\left(R_{0}+R_{c}\right) I_{0}-L_{0} \frac{d I_{0}}{d t}=0
$$

where $\Psi$ is the interlinkage flux of the winding. Vo is the terminal voltage of the power source. I $o$ is the current in the exciting winding. $R c$ is the dc resistance of the winding. Ro and $L, o$ are the resistance and the inductance of the lead wire and the load which are not included in the finite element region as shown in Fig.1.

The current density $\bar{J}$ in Eq.(1) is represented as follows $[1]$ :

$$
\mathbb{J}_{0}=\frac{\mathrm{n}_{\mathrm{C}}}{\mathrm{Sc}} I_{0}(\mathrm{i} \sin \theta \cos \psi+\mathrm{j} \sin \theta \sin \psi+\mathrm{k} \cos \theta)
$$

where $S c$ and $n c$ are the cross-sectional area and the number of turns of the winding respectively. $i, j$ and $k$ are the unit vectors in the $x^{-}, y-$ and $z$-directions respectively. $\psi$ and $\theta$ are the angles from the $x^{-}$and z-axes as shown in Fig. 2 .

The interlinkage flux $\Psi$ in Eq. (3) can be denoted by the $x^{-}, y^{-}$and $z$-components $A x, A y$ and $A z$ of $A$ in the winding as follows[1]:

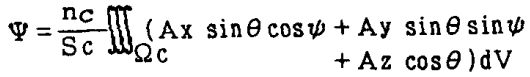

\subsection{Time-Periodic Finite Element Method}

When the waveform of a vector potential is symmetric and periodic with time as shown in Fig. 3 , the following relationship is hold between vector potentials $A^{t}$ and $A^{t+T / 2}$ at the instants $t$ and $\mathrm{t}+\mathrm{T} / 2(\mathrm{~T}:$ period):

$$
A^{t}=-A^{t+T / 2}
$$

The same relationship is hold on the current 10 as follows:

$$
I 0^{t}=-I 0^{t+T / 2}
$$

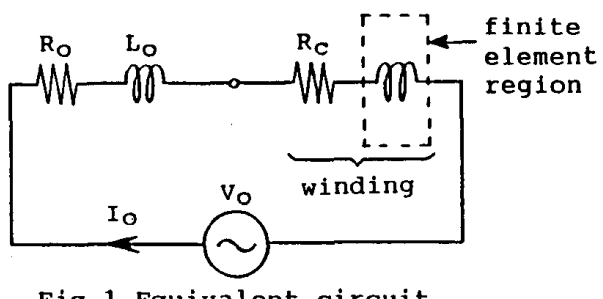

Fig.1 Equivalent circuit.

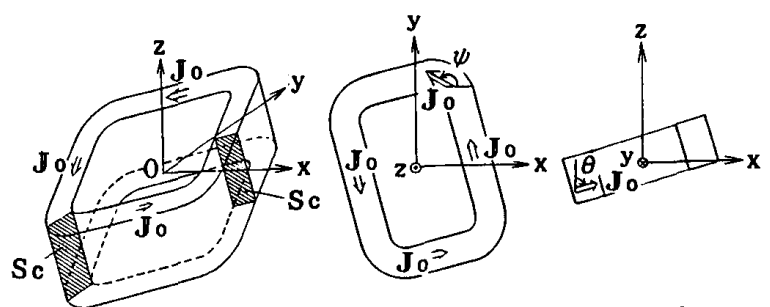

(a) bird's eye view (b) xy plane (c) $z x$ plane Fig. 2 winding. 
In the time-periodic finite element method, the vector potentials $A^{t}, A^{t+\Delta t},--, A^{t+T / 2-\Delta t} \quad(\Delta t$ : time interval) and the currents $1 o^{t}, \quad \mathrm{I} \mathrm{o}^{\mathrm{t}+\Delta t},-\cdots$, Io ${ }^{t+T / 2-\Delta t}$ are treated as unknown variables, and they are calculated simultaneously taking into account the relationships of Eqs.(6) and (7).

When the potential and the current at each instant are treated as unknown variables, the following equations for the non-linear analysis are obtained from Eqs. (1) to (5)|1]:

$$
\begin{aligned}
& {\left[\mathbf{C}^{t}\right]\left\{\begin{array}{l}
\delta u^{t-\Delta t} \\
\delta I 0 l^{t-\Delta t}
\end{array}\right\}+\left[\mathbb{H}^{t}\right]\left\{\begin{array}{l}
\delta u_{j}^{t} \\
\delta I O l^{t}
\end{array}\right\}} \\
& =\left\{\begin{array}{l}
-\left\{\mathbb{G}^{t}\right\} \\
-\left\{\eta r^{t}\right\}
\end{array}\right\} \\
& {\left[\mathrm{C}^{t+\Delta t}\right]\left\{\begin{array}{l}
\delta \mathbf{u}_{i}{ }^{t} \\
\delta I 0 l^{t}
\end{array}\right\}+\left[\mathbb{H}^{t+\Delta t}\right]\left\{\begin{array}{l}
\delta \mathbf{u}_{j}{ }^{t+\Delta t} \\
\delta I 0 l^{t+\Delta t}
\end{array}\right\}} \\
& =\left\{\begin{array}{l}
-\left\{G_{i}{ }^{t+\Delta t}\right\} \\
-\left\{\eta_{r^{t}}{ }^{t+\Delta t}\right\}
\end{array}\right\} \\
& {\left[\mathrm{C}^{t+T, 2-\Delta t}\right]\left\{\begin{array}{l}
\delta \mathrm{u}_{i}{ }^{t+T / 2-2 \Delta t} \\
\delta I 0 \mathrm{l}^{t+T / 2-2 \Delta t}
\end{array}\right\}} \\
& +\left[H^{t+T \cdot z-\Delta t}\right]\left\{\begin{array}{l}
\delta u^{t+T / 2-\Delta t} \\
\delta I O Q^{t+T / 2-\Delta t}
\end{array}\right\} \\
& =\left\{\begin{array}{l}
-\left\{\mathbb{G}_{i}{ }^{t+T / 2-\Delta t}\right\} \\
-\left\{\eta_{x^{2}+T / 2-\Delta t}\right\}
\end{array}\right\} \\
& \text { ( i , j }=1,2, \cdots n u) \\
& (\mathrm{k}, \ell=1,2, \cdots \mathrm{n} i)
\end{aligned}
$$

where nu is the number of unknown nodes of which the potentials are unknown. $\mathrm{ni}$ is the number of currents.

$\delta$ means the increment of the unknown variable. \{ui\} is denoted as follows:

$$
\{u i\}=\{\text { Axi, Ayi, Azi, } \phi i\}\}^{\top}
$$

Increments of potentials $\{\delta a\}$ and currents $\{\delta 1$ o $\}$ at the instants $t-\Delta t, t,---, t+T / 2-\Delta t$ are unknown variables. [ $[\mathbb{C}]$ and $[\mathbb{H} \mid$ are nearly the same as those of the conventional step-by-step method 1,5$]$.

By applying the relationships of Eqs. (6) and (7) to $\left\{\delta \mathbf{u}^{t-\Delta t}\right\}$ and $\left\{\delta \mathrm{I} 0 \mathrm{Q}^{t-\Delta t}\right\}$ in $\mathrm{Eq} .(8)$, the following matrix equation is obtained:

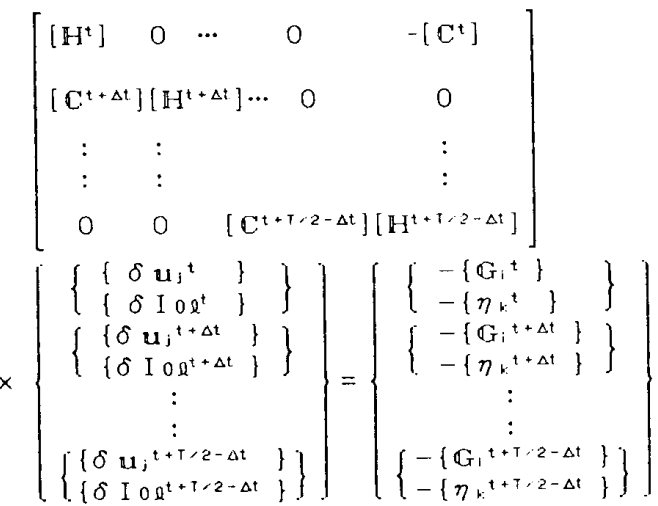

As the coefficient matrix in Eq. (10) is very large and non-symmetric, considerable CPU time and computer

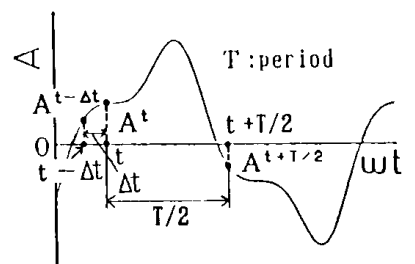

Fig. 3 periodic waveform.

storage are required using the conventional method of solution. Therefore, the iteration technique is introduced by dividing Eq. (10) into the following $\mathrm{m}$ equations:

$$
\begin{aligned}
& {\left[\mathbb{H}^{t+k \Delta t}\right]\left\{\begin{array}{l}
\delta \mathbf{u}^{t+k \Delta t} \\
\delta I 0 Q^{t+k \Delta t}
\end{array}\right\}} \\
& =-\alpha \cdot \beta_{k} \cdot\left[\mathrm{C}^{t+k \Delta t}\right]\left\{\begin{array}{l}
\delta \mathrm{u}^{\mathrm{t}+(k-1) \Delta t} \\
\delta \mathrm{I} 0 \mathrm{l}^{t+(k-1) \Delta t}
\end{array}\right\} \\
& +\left\{\begin{array}{l}
-\left\{\mathbb{G}_{i}{ }^{t+k \Delta t}\right\} \\
-\left\{\eta x^{t+k \Delta t}\right\}
\end{array}\right\} \\
& (\mathrm{k}=0,1, \cdots, \mathrm{m}-1)
\end{aligned}
$$

where $m$ is the number of time steps in half a period. $\beta \mathrm{k}$ is equal to $-1 \quad(k=0)$ and $1 \quad(k \neq 0) . \alpha$ is the relaxation factor and is chosen to be equal to 0 . The iteration is carried out until and io are converged. Using this iteration technique, the nonlinear steady state magnetic fields can be obtained within smaller CPU time than the conventional step-bystep method.

\section{AN EXAMPLE OH APPLICATION}

The currents in the primary and the secondary windings of a loaded transformer shown in Fig. 4 are analyzed. The core is made of non-oriented silicon steel M-15. The effective voltage and the frequency of the power source are $200 \mathrm{~V}$ and $50 \mathrm{~Hz}$ respectively. The numbers of turns of the primary and the secondary windings are both equal to 120 . Lo shown in Fig. 1 is assumed to be zero and $4 \Omega$ or $20 \Omega$ is selected for the load Ro.

Figures 5 and 6 show the current waveforms obtained by the step-by-step method and the timeperiodic method respectively.

Figure 7 shows the error of the current. The error $\varepsilon_{I}(k)$ is defined as follows:

$$
\varepsilon_{1}(x)=\frac{I \mathbb{m}^{(x)}-I m}{I m} \times 100 \%
$$

where $I m$ is the peak value of the current calculated by the time-periodic method. $1 \mathrm{~m}^{(\mathrm{k})}$ is the peak value of the current in the $k-t h$ half a period of the waveform calculated by the step-by-step method. The curves for $\varepsilon_{I}(k)>0$ and $\varepsilon_{I}(k)<0$ denote the errors for the even and the odd half a period

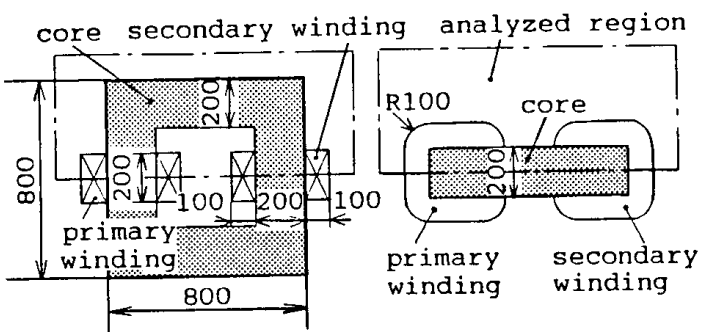

$\begin{array}{ll}\text { (a) front view (b) plan view } & \text { (b) }\end{array}$ Fig. 4 Analyzed model. 
respectively. Assuming that the exact solution is obtained when the error $\varepsilon_{1}(\mathrm{k})$ becomes less than $1 \%$, the number of iterations required for the step-bystep method is 3 to 6 periods in those examples.

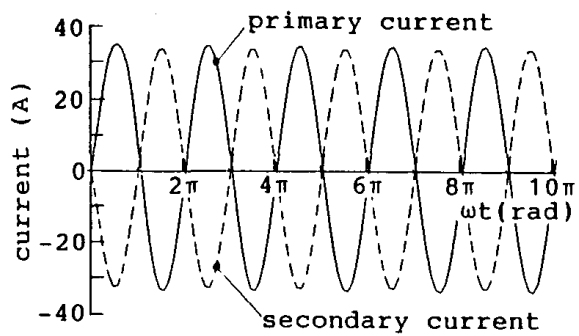

(a) $\mathrm{Ro}_{0}=4(\Omega)$

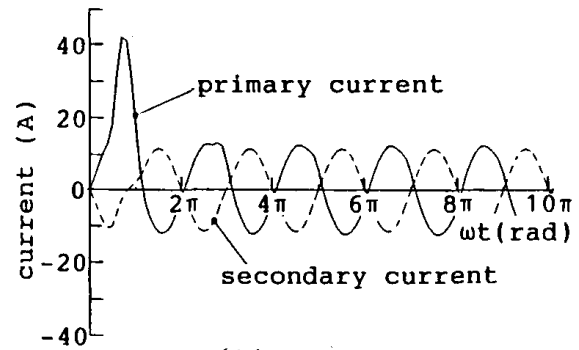

(b) $\mathrm{Ro}_{0}=20(\Omega)$

Fig. 5 Current waveforms (step-by-step method).

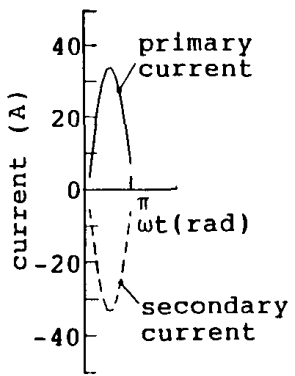

(a) $\mathrm{R}_{0}=4(\Omega)$

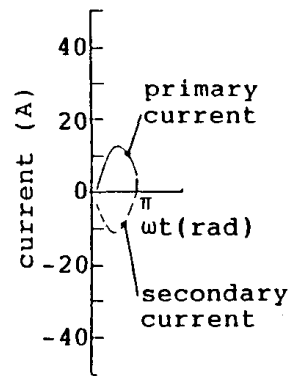

(b) $\mathrm{R}_{0}=20(\Omega)$
Fig. 6 Current waveforms (time-periodic method).

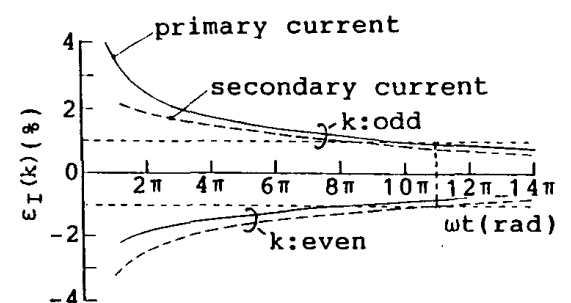

(a) $\mathrm{Ro}_{0}=4(\Omega)$

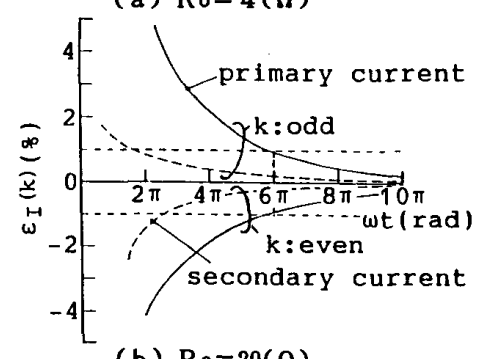

(b) $\mathrm{Ro}_{0}=20(\Omega)$

Fig. 7 Error of the current.
Table 1 denotes the comparison of the CPU time. The calculation of the step-by-step method is stopped when the error $\varepsilon I^{(k)}$ becomes less than $1 \%$. The CPU time of the time-periodic method can be reduced to about $1 / 3$ of the step-by-step method.

Table 2 denotes the computer storage $M . M$ is defined as the size of the dimensions declared in the computer code. The computer storage of the timeperiodic method is increased than that of the step-bystep method, because all unknown variables in half a period are memorized in the time-periodic method. However, the increase can be negligible.

Table 1 Comparison of CPU time

\begin{tabular}{c|c|c}
\hline \multirow{2}{*}{ method } & \multicolumn{2}{|c}{ CPU time (sec) } \\
\cline { 2 - 3 } & $\mathrm{R}_{0}=4(\Omega)$ & $\mathrm{R}_{0}=20(\Omega)$ \\
\hline step-by-step method & 446 & 298 \\
\hline time-periodic method & 139 & 103 \\
\hline
\end{tabular}

Table 2 Comparison of computer storage

\begin{tabular}{c|c}
\hline method & $M$ (MB) \\
\hline step-by-step method & 0.44 \\
\hline time-periodic method & 0.50 \\
\hline
\end{tabular}

\section{CONCLUSIONS}

The method for analvzing 3-D time-periodic nonlinear magnetic fields with eddy currents, in the case when electrical machines are excited from voltage sources, has been developed. It is shown that the CPU time can be reduced to, for example, about $1 / 3$ of the step-by-step method.

\section{ACKNOWLEDGEMENT}

This work was partly supported by the Grant-in-Aid for Developmental Scientific Research from the Ministry of Education, Science and Culture in Japan (No.62850046).

\section{REFERENCES}

[1] T.Nakata, N.Takahashi, K.Fu.jiwara and A.Ahagon : "3-D Finite Element Method for Analyzing Magnetic Fields in Electrical Machines Excited from Voltage Sources", IEEE Trans. Magnetics, MAG-24, 6, 2582 (1988).

121 T.Nakata and Y.Kawase: "Numerical Analysis of Nonlinear Transient Magnetic Field by Using the Finite Element. Method", Journal of IEE, Japan, 104B, $6,380(1984)$.

(3) T.Hara, T.Naito and J.Umoto : "Fjeld Analysis of Corona Shield Region in High Voltage Rotating Machines by Time-Periodic Finite Element Method: I. Numerical Calculation Method", ibid., 102-B, 7 , 423 (1982).

141 T.Nakata, N.Takahashi and Y.Kawase: "Non-Linear Analysis of Eddy Current Problems Using the TimePeriodic Finite Element Method", Proceedings of International Symposium on Electromagnetic Fields in Electrical Engineering, 89 (1985).

|5| T.Nakata, N.Takahashi, K.Fu.jiwara and M.Miura : "Finite Element Anaiysis of Nonlinear 3-D Magnetic Fields with Eddy Currents Using Magnetic Vector Potentials", Papers of Technical Meeting on Rotating Machinery and Static Apparatus, IEE of Japan, RM-86-39, SA-86-32 (1986). 